\title{
miR-140-5p regulates cell migration and invasion of non-small cell lung cancer cells through targeting VEGFA
}

\author{
PEIXIA YANG ${ }^{1}$, JIE XIONG $^{1}$, LIN ZUO $^{2}$, KEQUN LIU $^{3}$ and HOUBIN ZHANG ${ }^{4}$ \\ ${ }^{1}$ Respiratory Department of Internal Medicine, Linyi Central Hospital; ${ }^{2}$ Department of Internal Medicine, \\ Health Service Center of Lanshan District; ${ }^{3}$ Department of Hemodialysis, People's Hospital of Yishui County; \\ ${ }^{4}$ Department of Thoracic Surgery, Linyi People's Hospital, Linyi, Shandong 276000, P.R. China
}

Received October 10, 2017; Accepted June 22, 2018

DOI: $10.3892 / \mathrm{mmr} .2018 .9291$

\begin{abstract}
Lung cancer is the most common type of cancer worldwide, the most prevalent form of which is non-small cell lung cancer (NSCLC). MicroRNAs (miRs) are involved in the progression of NSCLC; however, the specific function of miR-140-5p in NSCLC remains unclear. The present study demonstrated that miR-140-5p was downregulated in the tumor tissues of patients with NSCLC, and it was associated with a poor prognosis. Furthermore, miR-140-5p significantly suppressed cell migration and invasion of the NSCLC cell line A549. In addition, the direct regulatory effect of miR-140-5p on vascular endothelial growth factor-A (VEGFA) was predicted by TargetScan and verified using a luciferase reporter gene assay. The present study also hypothesized that miR-140-5p may inhibit the expression of phosphorylated-protein kinase B by targeting VEGFA. In conclusion, miR-140-5p may be a potential target for the development of anti-neoplastic therapies in lung cancer.
\end{abstract}

\section{Introduction}

Lung cancer is the most common type of cancer and the leading cause of cancer-associated mortality worldwide (1). Non-small cell lung cancer (NSCLC) is the most common form of lung cancer (2). Recent advances in the diagnosis and treatment of cancer have been achieved; however, the 5-year overall survival rate of NSCLC is still only $11 \%$ (3). Consequently, an in-depth analysis of the mechanisms underlying NSCLC development and progression is required.

MicroRNAs (miRNAs/miRs) are small non-coding RNAs, which negatively regulate the expression of target genes by binding to the $3^{\prime}$ untranslated region (3'UTR) (4). It has

Correspondence to: Dr Houbin Zhang, Department of Thoracic Surgery, Linyi People's Hospital, 27 Jiefang Road, Lanshan, Linyi, Shandong 276000, P.R. China

E-mail: zhanghoubinlyph@aliyun.com

Key words: microRNA-140-5p, vascular endothelial growth factor-A, lung cancer, cell migration, cell invasion previously been demonstrated that miRNAs serve key roles in the development and progression of numerous types of cancer, including lung cancer (5). A previous study suggested that miR-140-5p is involved in cancer progression (6). Furthermore, numerous studies regarding miR-140-5p have been performed; for example, miR-140-5p has been demonstrated to inhibit the growth of ovarian cancer by suppressing the expression of platelet-derived growth factor receptor A (7). miR-140-5p has also been reported to inhibit the invasion and angiogenesis of breast cancer by targeting vascular endothelial growth factor-A (VEGFA) (8). The present study aimed to investigate the precise molecular mechanism of miR-140-5p in NSCLC.

VEGF, which serves a major role in angiogenesis, is a dimeric glycoprotein secreted from numerous cell types, including cancer cells (9). VEGF is a member of the platelet-derived growth factor family, which includes VEGFA, VEGFB, VEGFC, VEGFD and VEGFE (10). Upregulation of the VEGFA gene has been identified as a poor prognostic element for tumor-free survival in osteosarcoma (11). Therefore, VEGFA may be considered a potential target for cancer therapy.

The results of the present study demonstrated that downregulation of miR-140-5p was associated with clinical grading and metastasis in lung cancer tissues. In addition, VEGFA was verified to be a direct target of miR-140-5p. These findings suggested that miR-140-5p may function as a tumor suppressor, which affects lung cancer cell migration and invasion by inhibiting the expression of VEGFA.

\section{Materials and methods}

Cell culture and transfection. The lung cancer cell line A549 was purchased from The Cell Bank of Type Culture Collection of Chinese Academy of Sciences (Shanghai, China) and cultured in Dulbecco's modified Eagle's medium (DMEM; Gibco; Thermo Fisher Scientific, Inc., Waltham, MA, USA) supplemented with $10 \%$ fetal bovine serum (Gibco; Thermo Fisher Scientific, Inc.), $100 \mu \mathrm{g} / \mathrm{ml}$ streptomycin and $100 \mathrm{U} / \mathrm{ml}$ penicillin (Gibco; Thermo Fisher Scientific, Inc.) at $37^{\circ} \mathrm{C}$ in a humidified atmosphere containing $5 \% \mathrm{CO}_{2}$.

For cell transfection, A549 cells were seeded into 6-well plates at a density of $2 \times 10^{5}$ cells/well. Briefly, a miR-140-5p mimic (5'-CAGUGGUUUUACCCUAUGGUAG-3'; 100 nM; Guangzhou RiboBio Co., Ltd., Guangzhou, China) or a 
negative control (NC) mimic (5'-CUCACCAAAAACCCU AUGGUAG-3'; 100 nM; Guangzhou RiboBio Co., Ltd.) and Lipofectamine ${ }^{\circledR} 2000$ (Invitrogen; Thermo Fisher Scientific, Inc.) were diluted in DMEM. Following this, the mixture was added to the 6-well plates to obtain a final concentration of $20 \mathrm{nmol} / 1$ of hsa-miR-140-5p mimics or miR-NC mimics, and subsequently incubated at $37^{\circ} \mathrm{C}$ for $48 \mathrm{~h}$.

Luciferase reporter assay. The potential targets of miR-140-5p were analyzed using TargetScan (http://www.targetscan. org/vert_71/). The 3'-UTR of VEGFA was amplified by polymerase chain reaction (PCR), which was inserted into a pGL3-basic (Promega Corporation, Madison, WI, USA) using PrimeSTAR Max DNA Polymerase (Takara Biotechnology Co., Ltd., Dalian, China) to obtain a VEGFA-3'-UTR reporter construct. The primer sequences used for the amplification of VEGFA were as follows: Forward, 5'-GCTCTAGAGAGCCTC CCTCAGGGTTTC-3' and reverse, 5'-GCTCTAGAAAGG AATGTGTGCTGGGGAG-3'. The thermocycling conditions used for PCR were are follows: Denaturation for $10 \mathrm{sec}$ at $98^{\circ} \mathrm{C}$; followed by 35 cycles of annealing for $10 \mathrm{sec}$ at $55^{\circ} \mathrm{C}$ and elongation for $10 \mathrm{sec}$ at $72^{\circ} \mathrm{C}$. A VEGFA-3'-UTR-mutant (mut) reporter construct was also obtained by mutating the complementary seed sequences in the miR-140-5p-binding region using the QuikChange Site-Directed Mutagenesis kit (Agilent Technologies, Inc., Santa Clara, CA, USA). A549 cells $\left(2.5 \times 10^{4}\right)$ were transfected with $0.1 \mu \mathrm{g}$ reporter construct (mut or wild type), $100 \mathrm{nM}$ miR-140-5p mimic or NC, and $5 \mathrm{ng}$ Renilla luciferase vector (phRL-TK; Promega Corporation) by Lipofectamine ${ }^{\circledR}$ 2000 (Invitrogen; Thermo Fisher Scientific, Inc.), according to the manufacturer's protocol. A total of $24 \mathrm{~h}$ post-transfection, luciferase activity was measured by Luminoskan Ascent (Thermo Labsystems, Helsinki, Finland) and a Dual-Luciferase Reporter Assay kit (Promega Corporation), according to the manufacturers' protocols. Luciferase activity was normalized to Renilla luciferase activity.

Tissue samples. Paired NSCLC and adjacent non-tumor lung tissues were obtained from 30 patients who were treated at Linyi People's Hospital (Linyi, China) during tumor resection between January 2013 and February 2015. None of patients had previously undergone treatment prior to tissue collection. Prior written informed consent was obtained from each subject. The present study was approved by the Ethics Committee of Linyi People's Hospital.

Reverse transcription-quantitative PCR (RT-qPCR). Total miRNA was extracted using the mirVana miRNA Isolation kit (Ambion; Thermo Fisher Scientific, Inc.), according to the manufacturer's protocol. Total RNA was prepared by TRIzol ${ }^{\circledR}$ (Invitrogen; Thermo Fisher Scientific, Inc.), according to the manufacturer's protocol. cDNA was prepared using the PrimeScript RT Reagent kit (Takara Biotechnology Co., Ltd.) according to the manufacturer's protocol. Subsequently, cDNA was used to detect mRNA expression levels by qPCR using the SYBR ${ }^{\circledR}$ Premix Ex Taq ${ }^{\mathrm{TM}}$ II Perfect Real time kit (Takara Biotechnology Co., Ltd.) and an ABI 7500 Real-time PCR system (Applied Biosystems; Thermo Fisher Scientific, Inc.). mRNA and miRNA expression levels were determined using the $2^{-\Delta \Delta \mathrm{Cq}}$ method (12). For the detection of miRNAs,
miRNA purification miRNeasy Mini kit (Qiagen $\mathrm{GmbH}$, Hilden, Germany) was used for the extraction of total miRNA. TransScript First-Strand cDNA Synthesis SuperMix (Beijing Transgen Biotech Co., Ltd., Beijing, China) was used to perform reverse transcription, SYBR-Green 2x Master Mix (Thermo Fisher Scientific, Inc.) and RT-PCR primer sets were used. qPCR was performed using a CFX96 ${ }^{\mathrm{TM}}$ real-time PCR detection system (Bio-Rad Laboratories, Inc., Hercules, CA, USA). U6 was used as an internal control for miR-140-5p. $\beta$-actin was used as an internal control for VEGFA. The thermocycling conditions used for qPCR were as follows: $4 \mathrm{~min}$ at $94^{\circ} \mathrm{C}$ (initial activation); followed by 40 cycles of $30 \mathrm{sec}$ at $94^{\circ} \mathrm{C}$ (denaturation), $30 \mathrm{sec}$ at $58^{\circ} \mathrm{C}$ (annealing) and $30 \mathrm{sec}$ at $72^{\circ} \mathrm{C}$ (extension). The primer sequences used were as follows: miR-140-5p forward, 5'-GAGTGTCAGTGG TTTTACCCT-3' and reverse, 5'-GCAGGGTCCGAGGTA TTC-3'; VEGFA forward, 5'-TTTCTGCTGTCTTGGGTG CATTGG-3' and reverse, 5'-ACCACTTCGTGATGATTC TGCCCT-3'; $\beta$-actin forward, 5'-TCAAGATCATTGCTCCTC CTG-3' and reverse, 5'-CTGCTTGCTGATCCACATCTG-3'; and U6 forward, 5'-TGCGGGTGCTCGCTTCGGCAGC-3' and reverse, 5'-CCAGTGCAGGGTCCGAGGT-3'.

Western blot analysis. A total of $48 \mathrm{~h}$ post-transfection, A549 cells were washed with PBS and proteins were extracted using radioimmunoprecipitation assay buffer (Beyotime Institute of Biotechnology, Haimen, China). Protein concentration was determined using the bicinchoninic acid protein assay (Pierce; Thermo Fisher Scientific, Inc.). Samples (15 $\mu \mathrm{g} / \mathrm{lane})$ were separated by $12 \%$ SDS-PAGE and transferred to polyvinylidene difluoride membranes. Subsequently, the membranes were blocked using 5\% non-fat milk at room temperature for $1 \mathrm{~h}$, and subsequently probed with the following primary antibodies at $4^{\circ} \mathrm{C}$ overnight: Anti-AKT (cat. no. ab81283; 1:1,000), anti-phosphorylated AKT (cat. no. ab38449; 1:1,000) and anti-GAPDH (cat. no. ab8245; 1:1,000; all Abcam, Cambridge, UK). Following this, membranes were incubated with goat anti-rabbit horseradish peroxidase-conjugated secondary antibodies (cat. no. sc2004; 1:5,000; Santa Cruz Biotechnology, Inc., Dallas, TX, USA) at $37^{\circ} \mathrm{C}$ for $1 \mathrm{~h}$. Finally, the blots were visualized using enhanced chemiluminescent reagents (Thermo Fisher Scientific, Inc.).

Wound-healing assay. A549 cells transfected with miR-140-5p and NC mimics were seeded into 6 -well plates at $1 \times 10^{5}$ cells/1, and were cultivated in DMEM supplemented with $1 \%$ fetal bovine serum (FBS; Gibco; Thermo Fisher Scientific, Inc.) for $6 \mathrm{~h}$ at $37^{\circ} \mathrm{C}$ to allow adherent growth. Subsequently, a scratch was made in the cell layer using a $10-\mu 1$ pipette tip. After washing with serum-free medium, A549 cells were cultured at $37^{\circ} \mathrm{C}$ in an atmosphere containing $5 \% \mathrm{CO}_{2}$ for $24 \mathrm{~h}$. The wound-healing ability of the cells was visualized under a light microscope and calculated by measuring the distance between the scratches, as follows: Mobility ratio=(initial scratch width-current scratch width)/initial scratch width.

Transwell assay. Cell invasion was measured using Transwell cell culture chambers (Corning Incorporated, Corning, NY, USA) coated with $10 \mu 1$ Matrigel (1:3; BD Biosciences, San Jose, CA, USA). Briefly, A549 cells $\left(5 \times 10^{5} / 200 \mu 1\right)$ transfected 
A

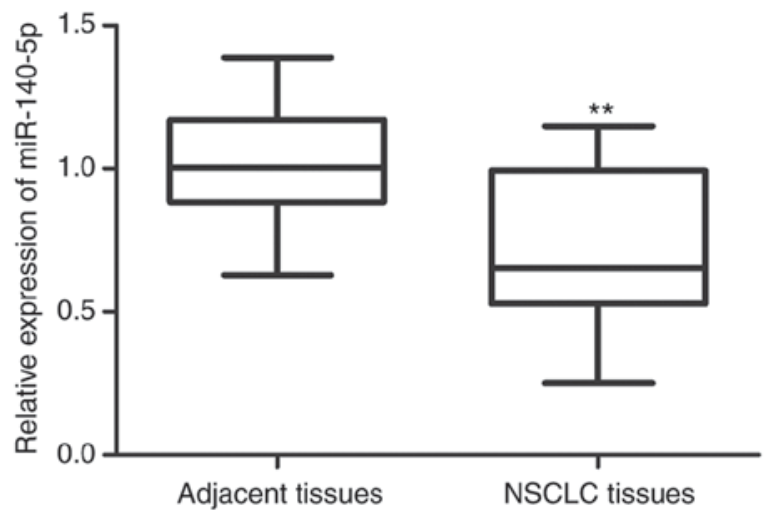

$B$

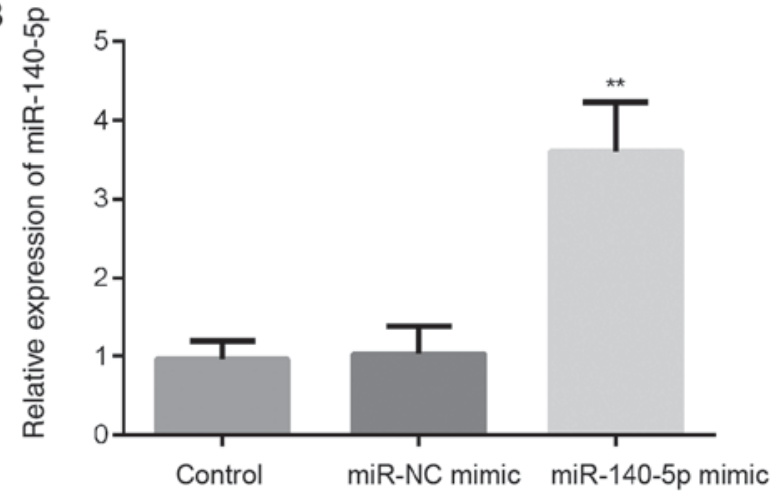

Figure 1. Loss of miR-140-5p in human NSCLC. (A) Reverse transcription-quantitative polymerase chain reaction indicated that miR-140-5p expression was significantly reduced in NSCLC tissues compared with in adjacent normal tissues. (B) miR-140-5p expression was significantly increased post-transfection of A549 cells with miR-140-5p mimic compared with in the control and miR-NC mimic cells. ${ }^{* *} \mathrm{P}<0.01$, NSCLC tissues vs. adjacent tissues and miR-140-5p mimic-transfected cells vs. control and miR-NC mimic-transfected cells. miR, microRNA; NC, negative control; NSCLC, non-small cell lung cancer.

with miR-140-5p and NC mimics were added to the upper chambers with complete culture medium, whereas serum-free medium with $5 \%$ FBS was added into the lower chambers. Following $24 \mathrm{~h}$ of incubation at $37^{\circ} \mathrm{C}$, cells that had invaded through the Matrigel were fixed with $4 \%$ paraformaldehyde for $30 \mathrm{~min}$ at room temperature and stained with $0.1 \%$ crystal violet at room temperature for $20 \mathrm{~min}$. Finally, cells within five fields of view were counted under a light microscope.

Statistical analysis. The quantitative values are presented as the means \pm standard error of the mean. The two-tailed Student's t-test was applied to compare between two groups. One-way analysis of variance followed by Student-Newman-Keuls post hoc analysis was applied to analyze the miR-140-5p expression among the $\mathrm{T}$ classification and $\mathrm{N}$ classification groups, as well as the differences between multiple groups. Statistical analyses were conducted using SPSS 18.0 (SPSS software, Inc., Chicago, IL, USA) and GraphPad Prism 5 (GraphPad Software, Inc., La Jolla, CA, USA). All experiments were repeated in triplicate. $\mathrm{P}<0.05$ was considered to indicate a statistically significant difference.

\section{Results}

Loss of miR-140-5p in human NSCLC is associated with patient N/M classification. To determine the expression levels of miR-140-5p in NSCLC, the expression levels of miR-140-5p were measured in 30 pairs of NSCLC samples and matched normal lung tissues by RT-qPCR. The results indicated that the miR-140-5p expression was significantly reduced in NSCLC tissues compared with in the matched normal tissues $(\mathrm{P}<0.01$; Fig. 1A). These findings are consistent with those of a previous study, which identified a decreased expression of miR-140 in NSCLC samples (13).

Furthermore, the association between miR-140-5p expression and the clinicopathological parameters of patients with NSCLC were analyzed and exhibited in Table I. Statistical analysis demonstrated that downregulation of miR-140-5p was significantly associated with $\mathrm{N}$ classification $(\mathrm{P}<0.001$, $\mathrm{f}=9.131)$ and $\mathrm{M}$ classification $(\mathrm{P}=0.002, \mathrm{t}=3.456)$; however, no significant association was observed with regards to the other parameters, including sex $(\mathrm{P}=0.432, \mathrm{t}=3.223)$, age $(\mathrm{P}=0.064$, $\mathrm{t}=1.932)$, smoking history $(\mathrm{P}=0.108, \mathrm{t}=1.663)$ or $\mathrm{T}$ classification $(\mathrm{P}=0.752, \mathrm{f}=0.403)$.

miR-140-5p has previously been demonstrated to be dysregulated in human colorectal cancer tissues (14), ovarian cancer tissues (7) and gastric cancer (15). In addition, miR-140-5p has been reported to inhibit cell invasion and migration $(14,16)$. Therefore, the following experiments were conducted to analyze the effects of miR-140-5p on NSCLC cell behavior, including cell invasion and migration.

The effects of the miR-140-5p mimic were initially detected on miR-140-5p expression in A 549 cells by RT-qPCR . The results demonstrated that, compared with in cells in the control and miR-NC mimic groups, the expression levels of miR-140-5p were significantly increased post-transfection with the miR-140-5p mimic ( $\mathrm{P}<0.01$; Fig. 1B).

miR-140-5p suppresses NSCLC cell migration in vitro. Using a wound-healing assay, it was demonstrated that overexpression of miR-140-5p significantly suppressed tumor cell migration in A549 cells compared with in the NC group ( $\mathrm{P}<0.01$; Fig. 2). This result suggested that miR-140-5p may suppress NSCLC cell migration in vitro.

miR-140-5p suppresses NSCLC cell invasion in vitro. Transwell assays with Matrigel revealed that miR-140-5p significantly decreased the invasive capacity of A549 cells $(\mathrm{P}<0.01$; Fig. 3). This result suggested that miR-140-5p may suppress NSCLC cell invasion in vitro.

miR-140-5p directly targets the 3'UTR of VEGFA. To elucidate the molecular mechanisms by which miR-140-5p performs its function, the potential targets of miR-140-5p were analyzed using computational methods including TargetScan. In particular, oncogenes that could be targeted by miR-140-5p were focused on. VEGFA was demonstrated to be a potential miR-140-5p target with a complementary sequence to miR-140-5p being identified in the 3'UTR of VEGFA by TargetScan analysis (Fig. 4A).

To confirm whether VEGFA is a direct downstream target of miR-140-5p, a luciferase reporter assay was performed. 
Table I. Basic characteristics of patients with non-small cell lung cancer.

\begin{tabular}{|c|c|c|c|c|c|}
\hline Characteristic & Case number & miR-140-5p expression & t-value & f-value & P-value \\
\hline Sex & & & 3.223 & & 0.432 \\
\hline Male & 20 & $0.741 \pm 0.066$ & & & \\
\hline Female & 10 & $0.655 \pm 0.071$ & & & \\
\hline Age (years) & & & 1.932 & & 0.064 \\
\hline$\geq 60$ & 19 & $0.700 \pm 0.068$ & & & \\
\hline$<60$ & 11 & $0.648 \pm 0.061$ & & & \\
\hline Smoking history & & & 1.663 & & 0.108 \\
\hline No & 11 & $0.692 \pm 0.098$ & & & \\
\hline Yes & 19 & $0.647 \pm 0.051$ & & & \\
\hline T classification & & & & 0.403 & 0.752 \\
\hline $\mathrm{T} 1$ & 9 & $0.648 \pm 0.065$ & & & \\
\hline $\mathrm{T} 2$ & 11 & $0.623 \pm 0.074$ & & & \\
\hline T3 & 8 & $0.633 \pm 0.047$ & & & \\
\hline $\mathrm{T} 4$ & 2 & $0.603 \pm 0.033$ & & & \\
\hline $\mathrm{N}$ classification & & & & 9.131 & $<0.001$ \\
\hline N0 & 11 & $0.776 \pm 0.087$ & & & \\
\hline N1 & 10 & $0.657 \pm 0.071$ & & & \\
\hline $\mathrm{N} 2$ & 6 & $0.601 \pm 0.065$ & & & \\
\hline N3 & 3 & $0.596 \pm 0.058$ & & & \\
\hline M classification & & & 3.456 & & 0.002 \\
\hline M0 & 25 & $0.703 \pm 0.091$ & & & \\
\hline M1 & 5 & $0.559 \pm 0.031$ & & & \\
\hline
\end{tabular}

T, tumor; N, node; M, metastasis.
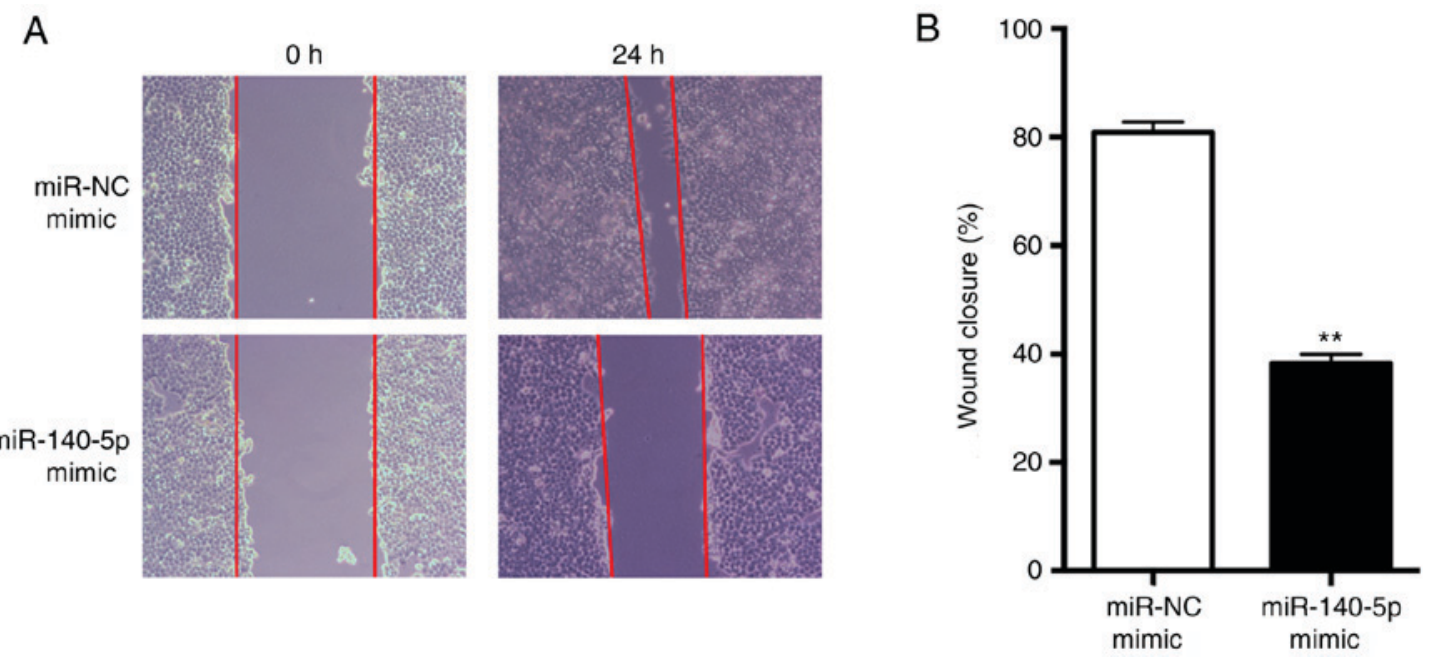

Figure 2. miR-140-5p inhibits non-small cell lung cancer cell migration in vitro. (A) Wound-healing assay demonstrated that transfection with the miR-140-5p mimic suppressed the migration of A549 cells compared with in the miR-NC mimic group (magnification, $\mathrm{x} 100)$. (B) Data were statistically analyzed. ${ }^{* *} \mathrm{P}<0.01$ miR-140-5p mimic-transfected cells vs. miR-NC mimic-transfected cells. miR, microRNA; NC, negative control.

The results demonstrated that overexpression of miR-140-5p significantly decreased the relative luciferase activity of VEGFA-3'UTR in A549 cells ( $\mathrm{P}<0.01$; Fig. 4B), but had no significant effect on the VEGFA-3'UTR mut. These results suggested that miR-140-5p may downregulate VEGFA expression by directly targeting its 3'UTR.
miR-140-5p inactivates $p$-AKT signaling by targeting and downregulating VEGFA. Finally, RT-qPCR analysis revealed that the average expression levels of VEGFA were significantly decreased in the miR-140-5p mimic group compared with in the NC group (Fig. 5A). These results indicated that VEGFA may be targeted by miR-140-5p in 
A

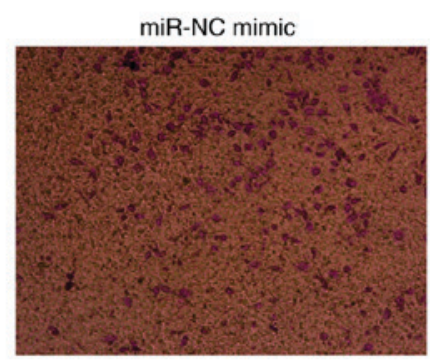

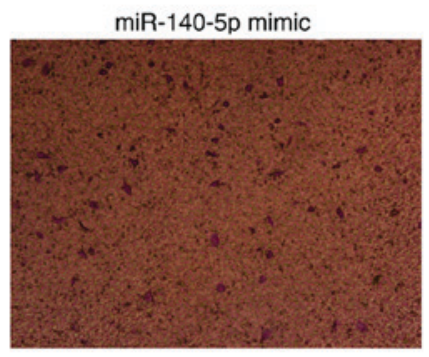

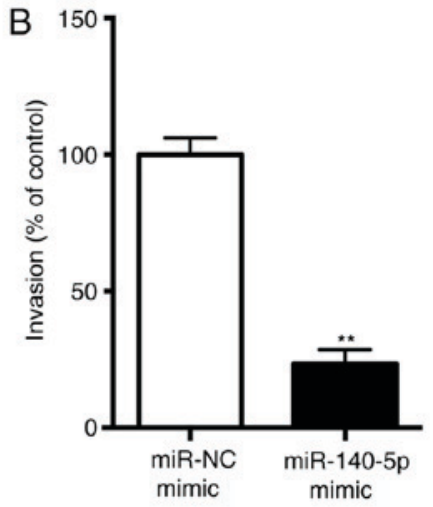

Figure 3. miR-140-5p inhibits non-small cell lung cancer cell invasion in vitro. (A) Using Transwell assays it was demonstrated that the miR-140-5p mimic decreased the invasive capacity of A549 cells (magnification, x100). (B) Data were statistically analyzed. ${ }^{* *} \mathrm{P}<0.01 \mathrm{miR}-140-5 \mathrm{p}$ mimic-transfected cells vs. miR-NC mimic-transfected cells. miR, microRNA; NC, negative control.

A $\begin{aligned} \text { Position 1063-1070 of VEGFA 3'UTR WT } & \text { 5'-UCCUCACACCAUUGAAACCACUA-3' } \\ \text { hsa-miR-140-5p } & 3^{\prime} \text {-GAUGGUAUCCCAUUUUGGUGAC-5' } \\ \text { VEGFA 3'UTR mut } & 5^{\prime} \text {-UCCUCACACCAUUGACCTTGGAA-3' }\end{aligned}$

B

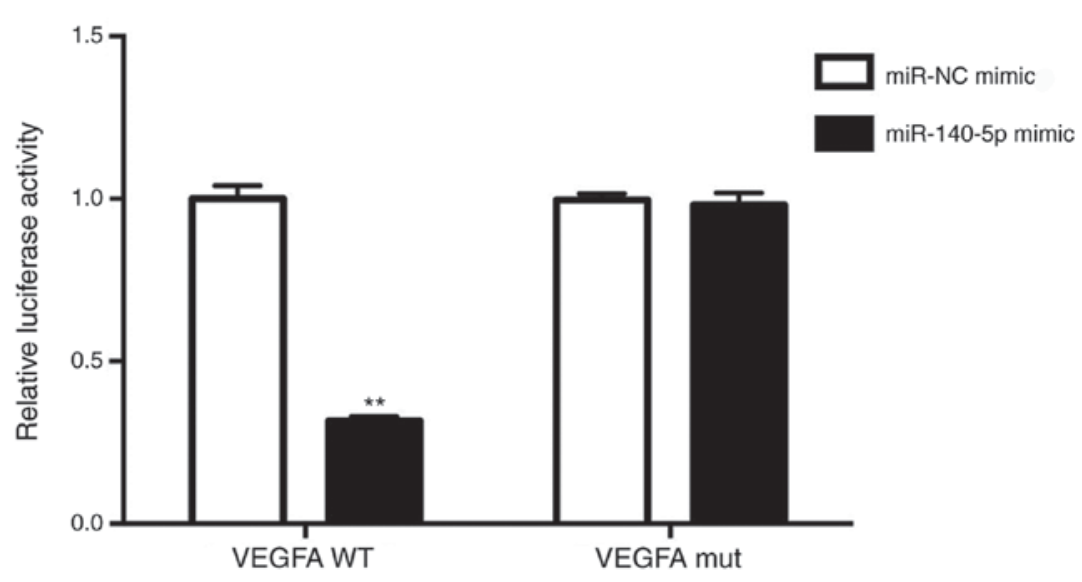

Figure 4. miR-140-5p directly targets the 3'UTR of VEGFA. (A) VEGFA 3'UTR was identified as a candidate target of miR-140-5p by TargetScan. (B) miR-140-5p mimic transfection significantly decreased the relative luciferase activity of VEGFA-3'UTR in A549 cells, but not of VEGFA-3'UTR mut. ${ }^{* *} \mathrm{P}<0.01$ miR-140-5p mimic-transfected cells vs. miR-NC mimic-transfected cells. miR, microRNA; mut, mutant; NC, negative control; UTR, untranslated region; VEGFA, vascular endothelial growth factor A; WT, wild type.

NSCLC A549 cells. Subsequently, the mechanisms by which the miR-140-5p/VEGFA axis affected the migration and invasion of NSCLC cells were investigated. The phosphatidylinositol 3-kinase/protein kinase B (PI3K/AKT) signaling pathway has been reported to be involved in angiogenesis and lung cancer progression (16). Therefore, the activation of p-AKT in NSCLC cells was investigated. Overexpression of miR-140-5p was observed to decrease the levels of p-AKT, with no obvious effects on the levels of total AKT (Fig. 5B). These results suggested that the miR-140-5p/VEGFA axis may negatively regulate the migration and invasion of NSCLC cells via $\mathrm{p}$-AKT signaling.

\section{Discussion}

NSCLC is the most common type of lung cancer, which is associated with a high morbidity and mortality rate worldwide (17).
The prognosis of patients with lung cancer is poor and treatment efficacy is not satisfactory (18). It is therefore necessary to develop novel therapeutic strategies for patients with NSCLC, and to perform an in-depth investigation into the development and progression of NSCLC.

It has previously been demonstrated that miRNAs serve key roles in the development and progression of lung cancer (5). The association between miR-140-5p and VEGFA has not been comprehensively investigated; however, miR-140-5p (13) and VEGFA $(19,20)$ have been implicated in NSCLC.

The present study demonstrated that the expression levels of miR-140-5p were decreased in NSCLC tissues compared with in matched normal tissues, which was consistent with a previous study (13). Furthermore, it was demonstrated that downregulation of miR-140-5p was significantly associated with the $\mathrm{N}$ classification and $\mathrm{M}$ classification of patients. These results indicated that miR-140-5p served a tumor suppressive 


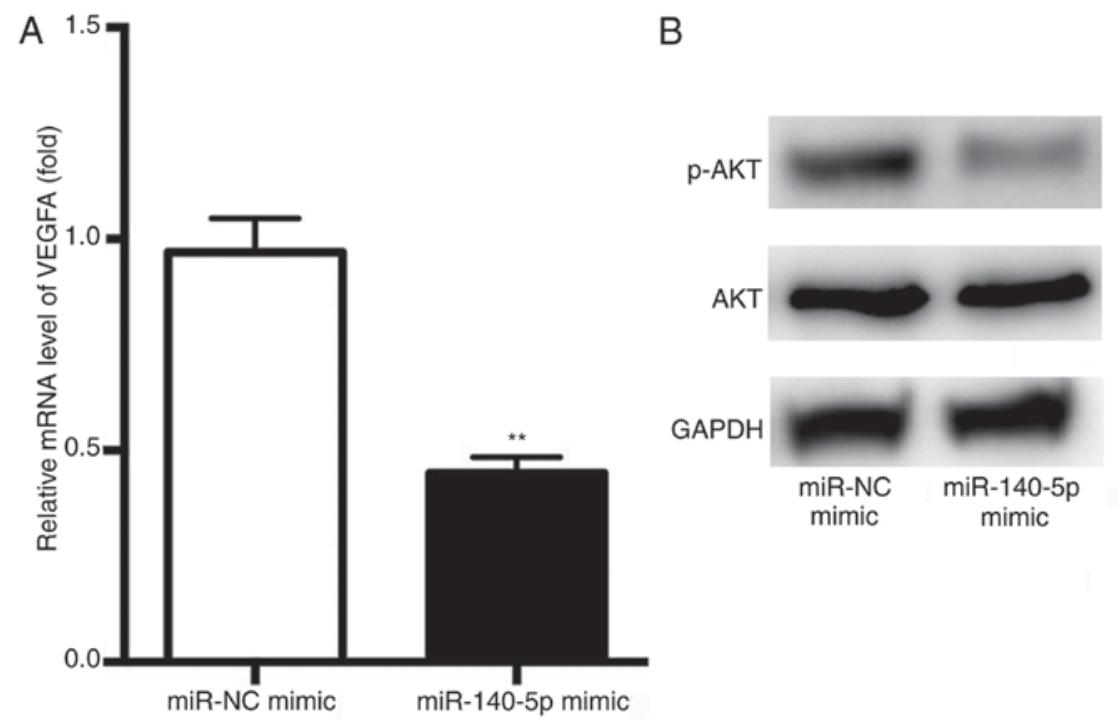

Figure 5. miR-140-5p inactivates p-AKT signaling by targeting and downregulating VEGFA. (A) Reverse transcription-quantitative polymerase chain reaction analysis revealed that the average expression level of VEGFA was significantly decreased in the miR-140-5p mimic group compared with in the NC group. (B) Transfection with the miR-140-5p mimic decreased the expression levels of p-AKT, but not AKT, compared with in the miR-NC mimic group. ${ }^{* *} \mathrm{P}<0.01$ miR-140-5p mimic-transfected cells vs. miR-NC mimic-transfected cells. AKT, protein kinase B; miR, microRNA; NC, negative control; p-, phosphorylated VEGFA, vascular endothelial growth factor A.

role in NSCLC; however, the effects of miR-140-5p on cell behavior remain to be investigated. Therefore, a miR-140-5p mimic was used to upregulate the expression levels of miR-140-5p in A549 cells, as verified by RT-qPCR.

NSCLC cell migration and invasion were demonstrated to be inhibited by overexpression of miR-140-5p, which is in agreement with the role of miR-140-5p in gastric cancer, as reported by Fang et al (15), who demonstrated that it significantly inhibited cell migration and invasion of AGS and BGC 823 cells. In addition, the function of miR-140-5p in breast cancer was demonstrated by Lu et al (8); miR-140-5p markedly suppressed the invasion of MCF-7 and MDA-MB-231 cells. However, to the best of our knowledge, it remains unknown how miR-140-5p executes its function in NSCLC.

The present study also aimed to determine the target mRNAs of miR-140-5p that functioned as oncogenes. VEGFA is essential for migration, invasion and angiogenesis in hepatocellular carcinoma (21), as well as tumor progression of NSCLC (22). Notably, VEGFA was identified as a candidate gene of miR-140-5p by TargetScan; this finding was validated by luciferase reporter assays in A549 cells.

Finally, the mechanisms by which the miR-140-5p/VEGFA axis affected the migration and invasion of NSCLC cells were investigated. The PI3K/AKT signaling pathway has been reported to serve a role in angiogenesis and lung cancer progression (16). As a crucial component of the PI3K/AKT signaling pathway, AKT mediates a large number of cellular responses through activating the expression of VEGFA $(23,24)$. A link between activation of the PI3K/AKT signaling pathway and increased expression of VEGF has been demonstrated in numerous studies $(25,26)$. Furthermore, VEGFA has been reported to induce AKT phosphorylation in NSCLC (22). Therefore, in the present study, the expression levels of VEGFA and p-AKT were analyzed by RT-qPCR and western blotting, respectively. Results revealed that the expression levels of VEGFA and p-AKT level were decreased in the miR-140-5p mimic group compared with in the NC group. Conversely, there were no obvious alterations in the protein expression levels of AKT between the miR-140-5p mimic and NC groups. These results suggested that the miR-140-5p/VEGFA axis may inhibit the migration and invasion of NSCLC cells via inactivation of p-AKT signaling. In conclusion, targeting miR-140-5p or VEGFA may serve as an appealing strategy for lung cancer therapy.

Activation of PI3K/AKT has previously been reported to stimulate VEGFA expression (27). In the present study, the results suggested that the miR-140-5p/VEGFA axis may inactivate p-AKT; however, several issues require further investigation: i) The association between VEGFA and p-AKT; ii) the association between p-AKT and miR-140-5p; and iii) the effects of VEGF on the PI3K/AKT signaling pathway, these issues will be investigated in future studies.

\section{Acknowledgements}

Not applicable.

\section{Funding}

No funding was received.

\section{Availability of data and materials}

The datasets used and/or analyzed during the current study are available from the corresponding author on reasonable request.

\section{Authors' contributions}

$\mathrm{HZ}$ designed the study, performed the data analyses and wrote the manuscript. PY, JX and LZ performed the experiments and analyzed the data. KL was responsible for patient enrollment, analyzed patient data and wrote the first draft of the manuscript prior to the re-editing of the manuscript by HZ. 


\section{Ethics approval and consent to participate}

The present study was approved by the Ethics Committee of Linyi People's Hospital. Prior written informed consent was obtained from each subject.

\section{Patient consent for publication}

Prior written informed consent was obtained from each subject.

\section{Competing interests}

The authors declare that they have no competing interests.

\section{References}

1. Molina JR, Yang P, Cassivi SD, Schild SE and Adjei AA Non-small cell lung cancer: Epidemiology, risk factors, treatment, and survivorship. Mayo Clin Proc 83: 584-594, 2008.

2. Siegel R, Ma J, Zou Z and Jemal A: Cancer statistics, 2014. CA Cancer J Clin 64: 9-29, 2014.

3. Verdecchia A, Francisci S, Brenner H, Gatta G, Micheli A, Mangone L and Kunkler I; EUROCARE-4 Working Group: Recent cancer survival in Europe: A 2000-02 period analysis of EUROCARE-4 data. Lancet Oncol 8: 784-796, 2007.

4. Bartel DP: MicroRNAs: Genomics, biogenesis, mechanism, and function. Cell 116: 281-297, 2004

5. Del Vescovo V, Grasso M, Barbareschi M and Denti MA: MicroRNAs as lung cancer biomarkers. World J Clin Oncol 5: 604-620, 2014

6. Ruan K, Fang X and Ouyang G: MicroRNAs: Novel regulators in the hallmarks of human cancer. Cancer Lett 285: 116-126, 2009.

7. Lan H, Chen W, He G and Yang S: miR-140-5p inhibits ovarian cancer growth partially by repression of PDGFRA. Biomed Pharmacother 75: 117-122, 2015.

8. Lu Y, Qin T, Li J, Wang L, Zhang Q, Jiang Z and Mao J: MicroRNA-140-5p inhibits invasion and angiogenesis through targeting VEGF-A in breast cancer. Cancer Gene Ther 24: 386-392, 2017.

9. Petrovic N: Targeting angiogenesis in cancer treatments: Where do we stand? J Pharm Pharm Sci 19: 226-238, 2016.

10. Shinkaruk S, Bayle M, Laïn G and Déléris G: Vascular endothelial cell growth factor (VEGF), an emerging target for cancer chemotherapy. Curr Med Chem Anticancer Agents 3: 95-117, 2003.

11. Yang J, Yang D, Sun Y, Sun B, Wang G, Trent JC, Araujo DM, Chen $\mathrm{K}$ and Zhang W: Genetic amplification of the vascular endothelial growth factor (VEGF) pathway genes, including VEGFA, in human osteosarcoma. Cancer 117: 4925-4938, 2011.

12. Livak KJ and Schmittgen TD: Analysis of relative gene expression data using real-time quantitative PCR and the 2(-Delta Delta C(T)) method. Methods 25: 402-408, 2001.

13. Yuan Y, Shen Y, Xue L and Fan H: miR-140 suppresses tumor growth and metastasis of non-small cell lung cancer by targeting insulin-like growth factor 1 receptor. PLoS One 8: e73604, 2013.
14. Zhai H, Fesler A, Ba Y, Wu S and Ju J: Inhibition of colorectal cancer stem cell survival and invasive potential by hsa-miR-140-5p mediated suppression of Smad2 and autophagy. Oncotarget 6: 19735-19746, 2015.

15. Fang Z, Yin S, Sun R, Zhang S, Fu M, Wu Y, Zhang T, Khaliq J and Li Y: miR-140-5p suppresses the proliferation, migration and invasion of gastric cancer by regulating YES1. Mol Cancer 16: $139,2017$.

16. Jeannot V, Busser B, Brambilla E, Wislez M, Robin B, Cadranel J, Coll JL and Hurbin A: The PI3K/AKT pathway promotes gefitinib resistance in mutant KRAS lung adenocarcinoma by a deacetylase-dependent mechanism. Int J Cancer 134: 2560-2571, 2014.

17. Chen HY, Yu SL, Chen CH, Chang GC, Chen CY, Yuan A, Cheng CL, Wang CH, Terng HJ, Kao SF, et al: A five-gene signature and clinical outcome in non-small-cell lung cancer. N Engl J Med 356: 11-20, 2007.

18. Jemal A, Bray F, Center MM, Ferlay J, Ward E and Forman D: Global cancer statistics. CA Cancer J Clin 61: 69-90, 2011.

19. Zhao D, Pan C, Sun J, Gilbert C, Drews-Elger K, Azzam DJ, Picon-Ruiz M, Kim M, Ullmer W, El-Ashry D, et al: VEGF drives cancer-initiating stem cells through VEGFR-2/Stat3 signaling to upregulate Myc and Sox2. Oncogene 34: 3107-3119, 2014.

20. Gu A, Lu J, Wang W, Shi C, Han B and Yao M: Role of miR-497 in VEGF-A-mediated cancer cell growth and invasion in non-small cell lung cancer. Int J Biochem Cell Biol 70: 118-125, 2016.

21. Ghosh A, Dasgupta D, Ghosh A, Roychoudhury S, Kumar D, Gorain M, Butti R, Datta S, Agarwal S, Gupta S, et al: MiRNA199a-3p suppresses tumor growth, migration, invasion and angiogenesis in hepatocellular carcinoma by targeting VEGFA, VEGFR1, VEGFR2, HGF and MMP2. Cell Death Dis 8: e2706, 2017.

22. Geng J, Li X, Zhou Z, Wu CL, Dai M and Bai X: EZH2 promotes tumor progression via regulating VEGF-A/AKT signaling in non-small cell lung cancer. Cancer Lett 359: 275-287, 2015.

23. Osaki M, Oshimura M and Ito H: PI3K-Akt pathway: Its functions and alterations in human cancer. Apoptosis 9: 667-676, 2004.

24. Shiojima I and Walsh K: Role of Akt signaling in vascular homeostasis and angiogenesis. Circ Res 90: 1243-1250, 2002

25. Hudson C, Liu M, Chiang G, Otterness D, Loomis D, Kaper F, Giaccia A and Abraham R: Regulation of hypoxia-inducible factor 1alpha expression and function by the mammalian target of rapamycin. Mol Cell Biol 22: 7004-7014, 2002.

26. Liu GT, Chen HT, Tsou HK, Tan TW, Fong YC, Chen PC, Yang WH, Wang SW, Chen JC and Tang CH: CCL5 promotes VEGF-dependent angiogenesis by down-regulating miR-200b through PI3K/Akt signaling pathway in human chondrosarcoma cells. Oncotarget 5: 10718-10731, 2014.

27. Qi L, Zhu F, Li SH, Si LB, Hu LK and Tian H: Retinoblastoma binding protein 2 (RBP2) promotes HIF-1 $\alpha$-VEGF-induced angiogenesis of non-small cell lung cancer via the Akt pathway. PLoS One 9: e106032, 2014.

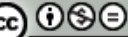

This work is licensed under a Creative Commons Attribution-NonCommercial-NoDerivatives 4.0 International (CC BY-NC-ND 4.0) License. 